\title{
Fixel-Based Analysis and Free Water Corrected DTI Evaluation of HIV Associated Neurocognitive Disorders
}

\author{
Alan Finkelstein ${ }^{1}$, Abrar Faiyaz ${ }^{2}$, Miriam T. Weber ${ }^{3}$, Xing Qiu ${ }^{4}$, Md Nasir Uddin 3,", Jianhui \\ Zhong $^{1,5,6}$, and Giovanni Schifitto ${ }^{2,3,6, *}$ \\ ${ }^{1}$ Department of Biomedical Engineering, University of Rochester, Rochester, NY, USA \\ ${ }^{2}$ Department of Electrical and Computer Engineering, University of Rochester, Rochester, NY, USA \\ ${ }^{3}$ Department of Neurology, University of Rochester, Rochester, NY, USA \\ ${ }^{4}$ Department of Biostatistics and Computational Biology, University of Rochester, Rochester, NY, \\ USA \\ ${ }^{5}$ Department of Physics and Astronomy, University of Rochester, Rochester, NY, USA \\ ${ }^{6}$ Department of Imaging Sciences, University of Rochester, Rochester, NY, USA
}

\section{*Correspondence to:}

Nasir_Uddin@URMC.Rochester.edu or Giovanni_Schifitto@URMC.Rochester.edu

Running Title: FBA and fwcDTI in HIV

Abstract Word Count: 200 words

Manuscript Word Count: 4,281 words (including in-text citations, but excluding the abstract, acknowledgements, reference list, as well as the table and figure legends)

Number of Primary Tables and Figures: 3 Table; 6 Figures

Number of Supplementary Materials: 1 Supplementary Tables, 2 Supplementary Figures

Total Page Count: 35 (including the abstract, manuscript body, figure captions, tables, references) 


\section{Abstract}

White matter damage is a consistent finding in HIV infected (HIV+) individuals. Previous studies have evaluated WM fiber tract specific brain regions in HIV-associated neurocognitive disorders using diffusion tensor imaging (DTI). However, DTI might lack an accurate biological interpretation, and the technique suffers from several limitations. Here, we sought to evaluate Fixel-based analysis (FBA) and free water corrected DTI (fwcDTI) metrics between HIV+ and HIV uninfected (HIV-) individuals, and their relationships with blood markers and cognitive scores. We also compared the specificity of both MRI metrics in their ability to distinguish between individuals with and without cognitive impairment using machine learning classifiers. Using 94 age-matched participants, we found that whole brain FBA was significantly reduced (up to 15\%) in various fiber bundles. Tract based special statistics (TBSS) of fwcDTI metrics revealed decreased fractional anisotropy FA (by $1-2 \%$ ) in HIV+ compared to HIV- individuals in areas consistent with those observed in FBA, but these were not significant. An adaptive boosting classifier reliably distinguished between cognitively normal patients and those with cognitive impairment with $80 \%$ precision and $78 \%$ recall. Therefore, FBA may serve as a potential in-vivo biomarker for evaluating and monitoring axonal degeneration in HIV+ patients at risk for neurocognitive impairment.

Keywords: HIV, Diffusion MRI, Fixel-Based Analysis, Free Water Imaging, Machine Learning, Cognitive Impairment.

\footnotetext{
Abbreviations: cART, Combined antiretroviral therapy; CNS, central nervous system; BBB, blood brain barrier; WM, white matter; HAND, HIV associated neurocognitive disorder; FW, free water; DTI, diffusion tensor imaging; FA, fractional anisotropy; AD, axial diffusivity; RD, radial diffusivity; MD, mean diffusivity; TBSS, Tract-based spatial statistics; CSF, cerebrospinal fluid; $\mathrm{FA}_{\mathrm{T}}$, free water corrected $\mathrm{FA} ; \mathrm{MD}_{\mathrm{T}}$, free water corrected MD; FBA, fixel based analysis; FD, fiber density; FC, fiber bundle cross section; FDC, fiber density cross section; AFD, apparent fiber density; FOD, fiber orientation distribution; MT-CSD, multi tissue constrained spherical deconvolution; GM, grey matter; NfL, neurofilament light chain; VVL, viral load; GLM, general linear model; CFE, connectivity based fixel enhancement; FDR, false discovery rate; ROI, region of interest; TFCE, threshold-free cluster enhancement; WNL, within normal limits; ANI, asymptomatic neurological impairment; MND, minor neurocognitive impairment; CI, cognitively impaired; kPCA, kernel principal component analysis; LDA, linear discriminant analysis; PPV, positive predictive value; ROC, receiver operating characteristic; AUC, area under the curve; fwcDTI, free water corrected DTI; DWI, diffusion weighted imaging; PLIC, posterior limb of the internal capsule; MCP, middle cerebellar peduncles; SCR, superior corona radiata; CP, cerebellar peduncle; SLF, superior longitudinal fasciculus; AdaBoost, adaptive boosting; PRC, precision recall curve; DMN, default mode network, CST, corticospinal tract.
} 


\section{Introduction}

Combined antiretroviral therapy (cART) has reduced morbidity and mortality rates significantly in HIV infected (HIV+) individuals (1). However, the increased survival may be masking an increase in cognitive impairment (2), mediated by injury to the central nervous system (CNS) and disruption of the blood brain barrier (BBB) (3). The HIV reservoir in the CNS resides primarily in microglia and perivascular macrophages, resulting in chronic neuroinflammation (4). While the small pool of infected cells in the CNS can release neurotoxic viral proteins, Tat and gp120, the larger pool of activated glia cells is responsible for the release of cytokines which can induce neuronal injury and cell death (5). HIV associated oligodendrocyte injury results in demyelination and alterations in white matter (WM) structural integrity (6). Thus, damage to WM fibers is likely a key factor in cognitive impairment observed in HIV associated neurocognitive disorder (HAND) (7).

MR neuroimaging studies have sought to identify potential in vivo biomarkers to investigate CNS injury in the setting of HIV infection (8). Structural and functional MRI have helped elucidate how atrophy and aberrant network topology mediate cognitive decline in HIV infection $(9,10)$. Nonetheless, given the well-established presence of WM alterations in HIV infection, it is paramount to further characterize WM in HAND. However, during chronic neuroinflammation, there may be contributing vasogenic edema (11), confounding the interpretation of WM lesions. Accordingly, appropriate models that accurately account for free water $(\mathrm{FW})$ contamination are necessary to sufficiently evaluate WM structural integrity in HIV infection (12).

Due to its noninvasiveness, diffusion tensor imaging (DTI) has been widely used in clinical neuroimaging studies $(13,14)$. DTI metrics such as fractional anisotropy (FA), axial diffusivity (AD), radial diffusivity (RD) and mean diffusivity (MD) characterize the orientation and distribution of the random movements of water molecules, diffusion magnitude, diffusional directionality 
perpendicular to the axon, and diffusional directionality along the axon, respectively $(15,16)$. Previous studies have shown that FA is decreased in the posterior limb of internal capsule (PLIC), the corticospinal tract (CST), temporal and frontoparietal WM regions, whereas RD and MD were increased in bilateral CST, temporal and frontal WM regions in HIV-infected (HIV+) individuals (17-22). Decreased FA has also been observed in the superior longitudinal fasciculus (SLF) and was correlated with decreased memory and executive function in HIV+ subjects exhibiting HAND (23). Tract-based spatial statistics (TBSS) is a popular voxel-based method to analyze DTI metrics, which maps control and disease cohort FA images to a WM skeleton to improve correspondence between subjects (24). We have previously reported diffuse FA and MD abnormalities using TBSS in HIV+ individuals (25).

However, voxel based measures are often contaminated by extracellular free water (FW) (12). FW contamination in the diffusion signal is due to water molecules that are not restricted by their environment, such as the cerebrospinal fluid (CSF). Edema caused by stroke (26), brain tumors (12), or neuroinflammation can also contaminate WM voxels (27). Accordingly, FW contaminated voxels will fit more towards an isotropic tensor and exhibit decreased FA values, confounding the interpretation of the results. Previously, several studies have reported that FW correction enhances specificity of DTI metrics (28-31) and reduces test-retest reproducibility errors (32). However, the diffusion tensor model is limited in that it is not able to reliably model complex and crossing-fiber populations, which are present in up to $90 \%$ of WM voxels $(33,34)$. Furthermore, while TBSS of FW corrected FA $\left(\mathrm{FA}_{\mathrm{T}}\right)$ is likely to provide more reliable measures of WM integrity in HIV infection, it does not include orientation information.

Fixel-based analysis (FBA) is a recent technique that models individual fibers at the sub-voxel level, termed fixels, which allow tract-specific comparisons (35). FBA enables the characterization of multiple fiber populations within a voxel, circumventing interpretation issues that commonly arise 
with voxel-averaged measurements such as FA and MD. Moreover, FBA accounts for both macrostructural (fiber bundle) and microstructural (within voxel) changes within WM, providing a more comprehensive understanding of intra-axonal and fiber tract changes. Accordingly, FBA has been used in several neurological disorders including Parkinson's disease $(36,37)$, multiple sclerosis $(38,39)$, traumatic brain injury $(40,41)$, schizophrenia (41) and healthy aging (42). FBA can be used to estimate fiber density (FD) within a fiber bundle, the fiber bundle cross section (FC) or a combined measure, fiber density cross section (FDC). FD is related to the intra-axonal volume, and a corresponding decrease in FD may reflect axonal degeneration (35). FD or the apparent fiber density (AFD) is calculated from the fiber orientation distribution (FOD) which is estimated using multi tissue constrained spherical deconvolution (MT-CSD) (43). By modeling grey matter (GM), WM, and CSF separately, MT-CSD accounts for FW contamination and has been shown to have better test-retest reliability than traditional DTI metrics (44). FC reflects the cross-sectional area of a fiber bundle, perpendicular to the length axis, and is derived from the Jacobian of the non-linear transformation from subject space to template space. Decreases in FC may result from WM atrophy and loss of the extra-axonal space, while increases may reflect inflammation and an increase in inflammatory proteins (35). FDC accounts for both macroscopic and microscopic effects on fiber density.

The aim of this study was to refine our understanding of how WM structural integrity is affected in HIV-infected individuals, and if these changes were associated with cognitive performance in HAND, using two approaches, FBA and fwcDTI. Additionally, we investigated whether fiber tract degeneration was related to inflammatory blood markers NfL and Tau of HIV infection. Machine learning classification, using a set of binary classifiers was also performed to distinguish cognitively normal individuals from those with cognitive impairment in HIV+ individuals. 


\section{Materials and Methods}

\subsection{Study Participants}

Forty-two treatment-nai $\square$ ve HIV+ participants (4 females and 40 males; mean age \pm standard error, $\mathrm{SE}=34.48 \pm 1.95$ years, range 20-63 years) and 52 age-matched HIV uninfected (HIV-) participants (26 females and 26 males; mean age $\pm \mathrm{SE}=37.02 \pm 1.66$ years, range $18-63$ years) were enrolled in a study assessing the potential neurotoxicity of combination antiretroviral therapy treatment (cART) study at the University of Rochester Medical Center. All participants provided written informed consent before enrollment according to the institutional protocol and underwent clinical, laboratory and brain MRI exams. All experiments were performed in accordance with relevant guidelines and regulations. The data reported here reflect the baseline assessment of HIV+, cART naive individuals prior to starting cART. This time-point was chosen because it represents the clearest difference in cognitive performance within the HIV+ group. Details about study participants (including age, sex, clinical results) are provided in Table 1.

\subsection{Data Acquisition}

\subsubsection{Blood Sample}

Plasma levels of markers associated with neuroinflammation and neurodegeneration (Neurofilament light chain NfL, and Tau protein) were measured by Simoa assay via commercial lab, QuanterixTM (Lexington, MA, United States, https://www.quanterix.com/). Viral load (VL) from each HIV+ participant was measured via Roche COBAS 8800 System with a lower limit of detection of 20 copies/mL. CD4+ count was obtained via flow cytometric immunophenotyping at the Clinical Laboratory Improvement Amendments (CLIA), certified clinical lab at the University of Rochester. 


\subsubsection{Neuropsychological assessments}

The neurocognitive evaluation was performed by trained staff and supervised by a clinical neuropsychologist. Tests of Executive Function (Trailmaking Test Parts A \& B, Stroop Interference task), Speed of Information Processing (Symbol Digit Modalities Test and Stroop 2 Color Naming), Attention and Working Memory (CalCAP(CRT4) and WAIS-III Letter-Number Sequencing), Learning (Rey Auditory Verbal Learning Test AVLT (trials 1-5), Rey Complex Figure Test Immediate Recall), Memory (Rey Auditory Verbal Learning Test RAVLT Delayed Recall, Rey Complex Figure Test Delayed Recall) and Motor (Grooved Pegboard, left and right hand)were administered at each visit. Premorbid intellectual functioning ability was estimated via WRAT-4 Reading at the baseline visit only. Raw scores were converted to z-scores using test manual norms. Cognitive domain scores were created by averaging the z-scores of tests within each domain. A total summary score was calculated by summing the z-scores of the six cognitive domains measured (Executive Function, Speed of Information Processing, Attention and Working Memory, Learning, Memory, and Motor). HAND diagnoses were determined for each participant according to the Frascati criteria (45). Subjects were accordingly defined as either within normal limits (WNL), or cognitively impaired (CI) [i.e., subjects having asymptomatic neurological impairment (ANI) or minor neurocognitive impairment (MND)].

\subsubsection{Image Acquisition}

All participants were scanned on a 3T MRI scanner ((MAGNETOM Trio, Siemens, Erlangen, Germany) equipped with a 32-channel head coil.

Anatomical Imaging: For the purpose of segmentation and identification of the anatomical landmarks, a T1-weighted (T1w) images were acquired using a 3D magnetization prepared rapid acquisition gradient-echo (MPRAGE) sequence with Inversion Time $(\mathrm{TI})=1,100 \mathrm{~ms}$, Repetition 
Time $(\mathrm{TR})=2,530 \mathrm{~ms}$, Echo Time $(\mathrm{TE})=3.44 \mathrm{~ms}$, Flip Angle $=7 \square$, Field of View $(\mathrm{FOV})=$ $256 \times 256$; GRAPPA factor $=2$, number of average $=1$, number of slices $=192$, voxel size $=$ $1.0 \times 1.0 \times 1.0 \mathrm{~mm}^{3}$, and total time of acquisition (TA) was 5:52 $\mathrm{min}$.

Diffusion Tensor Imaging: Diffusion weighted images (DWI) were acquired using a single shot spin echo echo-planar imaging (SE-EPI) sequence with 60 non-collinear diffusion-encoded images (b $\left.=1000 \mathrm{~s} / \mathrm{mm}^{2}\right), 10$ non-diffusion weighted reference images $\left(\mathrm{b}=0 \mathrm{~s} / \mathrm{mm}^{2}\right) ; \mathrm{TR}=8,900 \mathrm{~ms} ; \mathrm{TE}=86$ $\mathrm{ms} ; \mathrm{FOV}=256 \times 256 ;$ GRAPPA factor $=2 ;$ number of slices $=70 ;$ number of volumes $=61 ;$ voxel size $=2.0 \times 2.0 \times 2.0 \mathrm{~mm}^{3} ; \mathrm{TA}=10: 51 \mathrm{~min}$. In order to correct for EPI distortions, a double-echo gradient echo field map sequence was also acquired $(\mathrm{TR}=400 \mathrm{~ms}$; TE $=5.19 \mathrm{~ms}$; FOV $=256 \times 256$; flip angle $=60 \square$; number of slices $=70$; voxel size $=2.0 \times 2.0 \times 2.0 \mathrm{~mm}^{3} ; \mathrm{TA}=3: 28 \mathrm{~min}$ ).

\subsubsection{Image Preprocessing}

All MRI images were visually inspected for any severe artifacts. DWI images were corrected for eddy current-induced distortion, susceptibility-induced distortion, and motion correction using TOPUP and EDDY tools in FSL (https://fsl.fmrib.ox.ac.uk/fsl/fslwiki/) $(46,47)$.

\subsubsection{Fixel Based Analysis}

Fixel-based analysis (FBA) was performed using the recommended pipeline in MRtrix3 (www.mrtrix.org, version 3.0.2) (35). Briefly, DWI images were up-sampled by a factor of 2 in all three dimensions using cubic b-spline interpolation. The fiber orientation distributions (FODs) within each voxel were computed using single-shell, 3-tissue constrained spherical deconvolution (SS3TCSD), using group averaged response functions for WM, GM and CSF (43). A study-specific template was then created by spatial normalization of subjects using symmetric diffeomorphic nonlinear transformation FOD-based registration (48). One group-averaged FOD template was created 
for cross-sectional analysis, including $20 \mathrm{HIV}+$ and $20 \mathrm{HIV}$ - individuals. The FOD image for each subject was then registered to the template using FOD-guided non-linear registration.

A tractogram was then generated using whole-brain probabilistic tractography on the FOD population template (49). Twenty million streamlines were generated and subsequently filtered to two million streamlines using spherical deconvolution informed filtering of tractograms (SIFT) to reduce reconstruction biases (50). Fixel specific measures of fiber density (FD) and fiber bundle cross-section (FC) were calculated within each voxel. The $\log$ of FC (logFC) was calculated to ensure FC values were centered around zero and normally distributed. A combine measure, FDC, was also generated by multiplying FD and $\log$ FC. Modified fixel-based metrics were also calculated for the major fiber bundles using the Johns Hopkins University (JHU) DTI-based WM atlas.

\subsubsection{DTI preprocessing}

DTI metrics (FA and MD) were computed using DTIFIT in FSL (51). Free water corrected DTI (fwcDTI) metrics $\left(\mathrm{FA}_{\mathrm{T}}\right.$ and $\left.\mathrm{MD}_{\mathrm{T}}\right)$ were computed with a bi-tensor model from the DWI using a previously described algorithm (52) and the processing was performed using Nextflow pipeline (53) with all software dependencies bundled in a Singularity Container (54).

\subsection{Statistical Analysis}

\subsubsection{Subjects Characteristics}

Differences in clinical parameters between HIV+ and HIV - cohorts at baseline were examined using two-way independent t-tests at the $\alpha=0.05$ significance level. Statistical analysis of demographic data was computed in R 3.6.2 (R Foundation for Statistical Computing, Vienna, Austria).

Univariate comparisons between two independent groups were conducted by either two-group Welch's unequal variances t-test (for continuous variables) or Fisher exact test (for categorical 
variables). Paired t-tests were used to compare the levels of continuous variables in HIV+ participants between baseline and week-12. Pearson correlation test was used to test the univariate associations between two continuous variables. A p-value $<0.05$ was considered statistically significant for a single hypothesis testing problem. For inferential problems that involved multiple hypotheses, Benjamini-Hochberg multiple testing procedure was used to control the false discovery rate (FDR) at $\alpha<0.05$ level (55).

\subsubsection{Fixel-based analysis}

\subsubsection{Whole-brain fixed based analysis}

Statistical analyses of images were performed in MRtrix3 (www.mrtrix.org, version 3.0.2). All WM fixels were compared between HIV+ and HIV- individuals. Group comparisons were performed for FD, $\log \mathrm{FC}$, and FDC at each fixel using a General Linear Model (GLM), with age and sex included as covariates. Connectivity based fixel enhancement (CFE) and non-parametric permutation testing over 5000 permutations were used to identify significant differences in fixel-based metrics (56). Family-wise error (FWE)-corrected p values are reported to account for multiple comparisons. Significant fixels (FWE corrected p-value < 0.05) were visualized using the mrview tool in MRtrix3. Fixels were mapped to streamlines of the template-derived tractogram, only displaying streamlines corresponding to significant fixels. Significant streamlines were colored by the effect size, presented as a percentage relative to HIV- individuals or by streamline direction (left-right: red, inferiorsuperior: blue, anterior-posterior: green).

\subsubsection{Region of interest analysis}

Region of interest (ROI) analysis was performed for fixel-based metrics (FD, logFC and FDC), DTI metrics (FA and MD), fwcDTI metrics $\left(\mathrm{FA}_{\mathrm{T}}\right.$ and $\left.\mathrm{MD}_{\mathrm{T}}\right)$ using the JHU DTI-based WM atlas. The following ROIs were included in the analyses: the left and right posterior limb of internal 
capsule (PLIC), the left and right superior corona radiata (SCR), the left and right cerebellar peduncles $(\mathrm{CP})$, the left and right inferior cerebellar peduncle (ICP), and the middle cerebellar peduncle (MCP). These regions were chosen a priori based on the findings from whole brain fixel-based analysis. The mean value for FD, logFC, and FDC was computed for each ROI and compared across groups. Correlation analyses were also performed to evaluate the relationship between fixel-based metrics and cognitive z-scores. Independent t-tests with multiple comparisons corrections were used to compare mean $\log \mathrm{FC}$, FD, and FDC between cohorts over the ROIs. Correlations were performed using the non-parametric Spearman's rho and a linear model with age and sex as covariates. Benjamini-Hochberg procedure was applied to control the FDR at $\alpha<0.05$ significance level.

\subsubsection{Tract Based Spatial Statistics (DTI and fwcDTI)}

FSL based TBSS was performed to investigate the FA, free water corrected $F A\left(F A_{T}\right), M D$ and free water corrected $\mathrm{MD}\left(\mathrm{FA}_{\mathrm{T}}\right)$ changes along WM tracts (24). Group comparisons were performed using FSL Randomize for 5000 permutations. Threshold-free cluster enhancement (TFCE) (57) was used for multiple comparison correction at the $\alpha=0.05$ significance level.

\subsubsection{Machine Learning Classification}

Machine learning classification was performed using FBA and fwcDTI metrics. Classifiers were implemented in scikit-learn (58). Instances were standardized prior to training and dimensionality reduction was performed using kernel principal component analysis (kPCA). Four binary classifiers were used to evaluate the specificity of both fixel based metrics and fwcDTI metrics in their ability to distinguish between WNL and CI. In this study we implemented random forest, naïve bayes, linear discriminant analysis (LDA), and adaptive boosting (AdaBoost) classifiers. All classifiers were optimized using a grid search algorithm with a five-fold cross-validation. Classifiers were evaluated 
using the weighted average (across classes) for precision, recall and f1-score. Precision, also known as the positive predictive value (PPV), is defined as the number of instances classified as positive, divided by the total number of positive (CI) instances. Recall, or sensitivity, is the number of instances accurately classified as positive (true positives), divided by the total number of instances classified as positive. The F-score is the harmonic mean of precision and recall. Receiver operating characteristic (ROC) curves and precision-recall curves were also evaluated to assess the performance of these classifiers. Results are reported as the average across five-folds.

\section{Results}

\subsection{Participant Characteristics}

Clinical characteristics, demographic information, and cognitive scores for HIV+ and HIVindividuals are presented in Table 1. HIV+ and HIV- cohorts did not significantly differ in age, or ethnicity. The total summary z-score, attention z-score, and motor z-score were found to be significantly lower in the HIV+ cohort $(\mathrm{p}<0.05)$.

\subsection{Whole-brain fixel-based analysis}

Whole-brain FBA is shown in Figure 1. Streamlines corresponding to significant fixels (FWE corrected p-value $<0.05)$ are represented as the percentage decrease in HIV+ individuals compared to HIV - individuals for FD, $\log F C$, and FDC. Macrostructural decreases (measured via $\log F C$ ) of up to $15 \%$ were observed along specific fiber tracts. Specifically, the PLIC and MCP were affected bilaterally. The right SCR was also affected. Similar findings were observed for FD, though much less pronounced. Moreover, decreases in FD were more localized to the PLIC. FDC exhibited similar patterns of micro- and macro-structural degeneration, with a larger effect size (Table 2). Compared to HIV- individuals, HIV+ individuals had a $35 \%$ decrease in FDC in the PLIC bilaterally as well as 
the right SCR. Figure 2 shows streamlines displayed and colored based on orientation for significant decreases in $\log \mathrm{FC}$ in HIV+ individuals. Figure 3 shows a coronal view of fiber tract specific significant fixels, and the inset shows zoomed in area indicating regions with crossing fibers around cerebellar peduncles (CP) and MCP.

\subsubsection{Region of interest analysis}

Table 2 lists the mean and standard errors for several ROIs between the participants for the FBA metrics FD, logFC, and FDC for HIV+ and HIV- individuals. Linear models were further implemented to evaluate the relationship between fixel-based metrics and cohorts, including age and sex as covariates. We found a significant reduction in several ROIs in FBA metrics in the HIV+ than HIV- individuals. Linear regression models comparing DTI and fwcDTI metrics between HIV+ and HIV - cohorts are provided in Supplementary Table 1. None of the ROIs show any significant differences between the cohorts for the DTI and fwcDTI metrics.

Figure 4(A-C) shows scatterplots examining the relationship between the attention domain zscores and FBA metrics (FD, $\log$ FC and FDC) for the right and left of PLIC and SCR. For HIV+ individuals, the right PLIC was found to be significant for FD $(\rho=0.33, p=0.036)$, FDC $(\rho=0.44$, $\mathrm{p}=0.0039)$ and $\log \mathrm{FC}(\rho=0.4, \mathrm{p}=0.0085)$ while the right and left SCR were found to be significant in FDC $(\rho=0.43, p=0.0048$, and $\rho=0.38, p=0.014$, respectively). On the other hand, none of the ROIs for any metrics were significantly correlated with attention z-scores in the HIV-individuals. Figure 4D illustrates the corresponding ROIs.

Additionally, the right PLIC was also found to be significantly correlated with tau protein in HIV+ individuals $(\rho=0.32, p=0.043)$ (Supplementary Figure S1). However, no significant associations were observed between FBA metrics and NfL. 


\subsection{Tract Based Spatial Statistics (DTI and fwcDTI)}

No significant differences between HIV+ and HIV- cohorts were observed in FA and FA $\mathrm{F}_{\mathrm{T}}$ using TBSS. However, a decrease in FA and $\mathrm{FA}_{\mathrm{T}}(1-2 \%)$ was observed in the PLIC, SCR and superior longitudinal fasciculus (SLF) using TBSS (Figure 5). TBSS of FA $\mathrm{F}_{\mathrm{T}}$, while not significant, highlighted areas that were decreased in HIV+ individuals compared to HIV- individuals. It should be noted that in our study, the TBSS figures (Figure 5) report regions found for $\boldsymbol{p}<0.5$. None of the regions survived for significance thresholding for $p<0.05$.

\subsection{Machine Learning Classification}

Overall, we found that use of fixel based metrics resulted in a higher precision and recall compared to when using fwcDTI metrics. The adaptive boosting (AdaBoost) method resulted in the best performance for fixel based metrics. Figure 6 shows the ROC and PRC curves using an AdaBoost multiclass classifier. Table 3 represents the sample averaged precision, recall and $\mathrm{f} 1$-scores for other classifiers contrasting specificity of FBA and fwcDTI using five-fold cross validation.

\section{Discussion}

In this study, we evaluated fiber tract-specific WM changes in HIV infection using FBA, DTI and fwcDTI metrics. The major findings of this work were that: a) HIV+ individuals exhibit axonal degradation within the PLIC, CP and SCR as revealed by FBA; b) Similar trends were observed using TBSS of FA and $\mathrm{FA}_{\mathrm{T}}$. In contrast to $\mathrm{FA}, \mathrm{FA}_{\mathrm{T}}$ showed trends towards more areas that were decreased in HIV+ individuals compared to HIV- individuals. c) FBA metrics in PLIC and SCR exhibit significant positive associations with attention cognitive z-scores in HIV+ individuals; d) Machine learning classifiers for FBA reliably distinguished between cognitively normal patients and those with cognitive impairment in patients with HIV infection. 
To the best of our knowledge, this is the first study investigating FBA and TBSS of fwcDTI metrics $\left(\mathrm{FA}_{\mathrm{T}}\right.$, and $\left.\mathrm{MD}_{\mathrm{T}}\right)$ in HIV infected individuals. The work presented here provides a comprehensive and robust framework for evaluating brain injury (and secondary chronic inflammation) in the setting of HIV. Chronic neuroinflammation results in damage to the CNS, alteration of the blood brain barrier (BBB), and chronic edema (59). Changes in whole-brain FBA were found along distinct fiber tracts associated with motor and attention cognitive domains. In particular PLIC, CP, MCP, and SCR were affected and exhibited reduced fiber density and fiber bundle cross-section in HIV+ individuals compared to HIV- individuals. ROI based analysis revealed lower mean fixel-based metrics in the HIV+ cohort compared to the HIV-cohort, consistent with those obtained from the whole-brain FBA results.

Previous work using DTI has shown that FA is decreased in corticospinal tract and that MD is increased in the corticospinal tract bilaterally (23). However, FW contamination results in fitting each voxel with an isotropic tensor, leading to an erroneous conclusion that FA is decreased in the presence of edema (12). Consistent with previous work, our findings suggest that axonal degeneration occurs only in fixels associated with the CST in HIV infection, but accounting for edema and FW contamination. Moreover, FBA is a more robust method to evaluate WM structural integrity compared to TBSS, with or without FW correction. This is likely because constrained spherical deconvolution estimates the response function separately for WM, GM, and extracellular FW, ultimately better modeling the response function of WM than DTI (44).

In addition, our findings are consistent with previous work investigating WM in HIV infection, and with the clinical presentation of HAND (2). However, the present study emphasizes FBA to provide a more robust means to evaluate WM structural integrity independent of partial volume effects and FW contamination. Although we only saw trends using TBSS of fwcDTI, it is reasonable to implement this approach to DTI data. Clinically, HAND is a spectrum of disorders in 
which patients may present with difficulties in cognition, particularly declines in psychomotor processing, attention, and memory. Of interest, the role of the corona radiata in motor pathways is well established, however recent studies have suggested that the corona radiata is related to attention as well (60). As a major WM intersection, it is possible that damage to the corona radiata, observed in this study, affects both corticospinal fibers as well as association fibers passing through the SCR, contributing to the diffuse cognitive changes seen in HAND, particularly psychomotor slowing. Additionally, the default mode network (DMN) has been implicated in HIV and HAND (9). The DMN is primarily composed of the medial prefrontal cortex, posterior cingulate cortex, precuneus and angular gyrus. Moreover, the DMN, a task-negative network is associated with attention and memory. Thus, it is feasible that degeneration of association fibers passing through the corona radiata and internal capsule disrupt connections to the $\mathrm{DMN}(61)$.

We also investigated the relationship between fixel based metrics (i.e., FD, $\operatorname{logFC}$ and FDC) and inflammatory blood markers including CD4, VL, and neuronal markers NfL, and tau protein in HIV+ individuals. The PLIC was the only structure significantly correlated with tau protein $(\rho=0.32$, $\mathrm{p}=0.043$ ). No other blood markers were significantly correlated with fixel-based metrics. Tau protein is a component of the neurofibrillary tangles most often associated with Alzheimer's Disease (62). However, increasing evidence suggests that chronic neuroinflammation in the setting of HIV infection predisposes HIV+ individuals to premature neurodegeneration as measured by tau protein (63).

Lastly, several machine learning classifiers were used to classify cognitive status in HIV+ individuals using fixel based and free water corrected DTI metrics. In general, we observed that fixel based metrics results in improved performance as measured with precision, recall and f1-score, compared to fwcDTI metrics. Inclusion of other relevant imaging metrics and biomarkers is likely to further improve prediction of developing HAND. 
This study has some limitations. First, only 6 of the subjects in the study had mild neurological disorder (MND), therefore our study was mostly composed of cognitively normal subjects and patients with ANI. In this work, MND and ANI were combined and categorized as CI. Second, the proportion of male and female subjects was not equal in HIV+ cohort. However, FBA, and DTI metrics were not significantly different in males vs. females in our HIV- participants, which had a more equal representation. Future research will investigate the utility of fixel based metrics in evaluating HIV associated neuroinflammation longitudinally and developing prognostic machine learning models for predicting HAND longitudinally.

\section{Conclusions}

Our findings suggest that FBA provides a comprehensive and accurate assessment of WM structural integrity in the setting of chronic neuroinflammation in HIV population. Our results indicate that degeneration occurs along specific fiber tracts, which manifests both as macrostructural and microstructural alterations; particularly in the internal capsule and corona radiata in HIV+ individuals. Moreover, our findings are consistent with the clinical presentation of HAND, which often presents as psychomotor slowing with impaired attention, memory, and fine motor function. TBSS based analysis of free water corrected and uncorrected DTI metrics showed decreasing trends between the HIV+ and control group. However, these were not significant, suggesting lower sensitivity for the level of pathology in the cohort under investigation compared to FBA. Therefore, FBA may provide a sensitive biomarker to monitor axonal degeneration in individuals with HIV infection. 


\section{Acknowledgments}

This work was supported by the National Institutes of Health (NIH) grants R01-MH099921, R01AG054328, and R01-MH118020. We thank our study participants and staff who were involved in image acquisition and clinical/neurocognitive assessments.

\section{Conflict of Interest}

The authors declare no conflicts of interests.

\section{Author Contributions}

AF (Alan Finkelstein): Study concept, image processing, data analysis and interpretation, manuscript writing, original draft; AF (Abrar Faiyaz): Image processing, data analysis and interpretation, and manuscript review for intellectual content; MTW: Cognitive data collection and manuscript review for intellectual content; XQ: Statistical interpretation, and manuscript review for intellectual content; MNU: Study concept and interpretation, manuscript writing and review for intellectual content; JZ: Manuscript review for intellectual content; GS: Project administration, funding acquisition, interpretation, manuscript review for intellectual content. All authors contributed to the article and approved the submitted version.

\section{Data Availability Statement}

Anonymized data will be made available on reasonable request, pending appropriate institutional review board approvals. 


\section{Approval for human experiments}

All protocols were reviewed and approved by the Research Subjects Review Board (RSRB) at the University of Rochester. All participants signed a written consent form and all experiments were performed in accordance with relevant guidelines and regulations.

\section{References}

1. Collaboration H-C, Ray M, Logan R, Sterne JA, Hernandez-Diaz S, Robins JM, Sabin C, Bansi L, van Sighem A, de Wolf F, Costagliola D, Lanoy E, Bucher HC, von Wyl V, Esteve A, Casbona J, del Amo J, Moreno S, Justice A, Goulet J, Lodi S, Phillips A, Seng R, Meyer L, PerezHoyos S, Garcia de Olalla P, Hernan MA. The effect of combined antiretroviral therapy on the overall mortality of HIV-infected individuals. AIDS. 2010;24(1):123-37. Epub 2009/09/23. doi: 10.1097/QAD.0b013e3283324283. PubMed PMID: 19770621; PMCID: PMC2920287.

2. Clifford DB, Ances BM. HIV-associated neurocognitive disorder. Lancet Infect Dis. 2013;13(11):976-86. Epub 2013/10/26. doi: 10.1016/S1473-3099(13)70269-X. PubMed PMID: 24156898; PMCID: PMC4108270.

3. Atluri VS, Hidalgo M, Samikkannu T, Kurapati KR, Jayant RD, Sagar V, Nair MP. Effect of human immunodeficiency virus on blood-brain barrier integrity and function: an update. Front Cell Neurosci. 2015;9:212. Epub 2015/06/27. doi: 10.3389/fncel.2015.00212. PubMed PMID: 26113810; PMCID: PMC4461820.

4. Bissel SJ, Wiley CA. Human immunodeficiency virus infection of the brain: pitfalls in evaluating infected/affected cell populations. Brain Pathol. 2004;14(1):97-108. Epub 2004/03/05. doi: 10.1111/j.1750-3639.2004.tb00503.x. PubMed PMID: 14997942; PMCID: PMC1449744. 
5. Yadav A, Collman RG. CNS inflammation and macrophage/microglial biology associated with HIV-1 infection. J Neuroimmune Pharmacol. 2009;4(4):430-47. Epub 2009/09/22. doi:

10.1007/s11481-009-9174-2. PubMed PMID: 19768553; PMCID: PMC5935112.

6. Liu H, Xu E, Liu J, Xiong H. Oligodendrocyte Injury and Pathogenesis of HIV-1-Associated Neurocognitive Disorders. Brain Sci. 2016;6(3). Epub 2016/07/28. doi: 10.3390/brainsci6030023. PubMed PMID: 27455335; PMCID: PMC5039452.

7. Alakkas A, Ellis RJ, Watson CW, Umlauf A, Heaton RK, Letendre S, Collier A, Marra C, Clifford DB, Gelman B, Sacktor N, Morgello S, Simpson D, McCutchan JA, Kallianpur A, Gianella S, Marcotte T, Grant I, Fennema-Notestine C, Group C. White matter damage, neuroinflammation, and neuronal integrity in HAND. J Neurovirol. 2019;25(1):32-41. Epub 2018/10/07. doi: 10.1007/s13365-018-0682-9. PubMed PMID: 30291567; PMCID: PMC6416232.

8. Ances BM, Hammoud DA. Neuroimaging of HIV-associated neurocognitive disorders (HAND). Curr Opin HIV AIDS. 2014;9(6):545-51. Epub 2014/09/25. doi: 10.1097/COH.0000000000000112. PubMed PMID: 25250553; PMCID: PMC4217490.

9. Zhuang Y, Qiu X, Wang L, Ma Q, Mapstone M, Luque A, Weber M, Tivarus M, Miller E, Arduino RC, Zhong J, Schifitto G. Combination antiretroviral therapy improves cognitive performance and functional connectivity in treatment-naive HIV-infected individuals. J Neurovirol. 2017;23(5):704-12. Epub 2017/08/10. doi: 10.1007/s13365-017-0553-9. PubMed PMID: 28791662; PMCID: PMC5655604.

10. Zhuang Y, Zhang Z, Tivarus M, Qiu X, Zhong J, Schifitto G. Whole $\square$ brain computational modeling reveals disruption of microscale brain dynamics in HIV infected individuals. Human brain mapping. 2021;42(1):95-109. 
11. Stamatovic SM, Dimitrijevic OB, Keep RF, Andjelkovic AV. Inflammation and brain edema: new insights into the role of chemokines and their receptors. Acta Neurochir Suppl. 2006;96:444-50. Epub 2006/05/05. doi: 10.1007/3-211-30714-1_91. PubMed PMID: 16671502.

12. Pasternak O, Sochen N, Gur Y, Intrator N, Assaf Y. Free water elimination and mapping from diffusion MRI. Magn Reson Med. 2009;62(3):717-30. Epub 2009/07/23. doi: 10.1002/mrm.22055. PubMed PMID: 19623619.

13. Basser PJ, Mattiello J, LeBihan D. MR diffusion tensor spectroscopy and imaging. Biophysical journal. 1994;66(1):259-67.

14. Le Bihan D, Mangin JF, Poupon C, Clark CA, Pappata S, Molko N, Chabriat H. Diffusion tensor imaging: concepts and applications. J Magn Reson Imaging. 2001;13(4):534-46. Epub 2001/03/29. doi: 10.1002/jmri.1076. PubMed PMID: 11276097.

15. Alexander AL, Lee JE, Lazar M, Field AS. Diffusion tensor imaging of the brain. Neurotherapeutics. 2007;4(3):316-29. Epub 2007/06/30. doi: 10.1016/j.nurt.2007.05.011. PubMed PMID: 17599699; PMCID: PMC2041910.

16. Beaulieu C. The basis of anisotropic water diffusion in the nervous system-a technical review. NMR in Biomedicine: An International Journal Devoted to the Development and Application of Magnetic Resonance In Vivo. 2002;15(7ロ8):435-55.

17. Nir TM, Jahanshad N, Busovaca E, Wendelken L, Nicolas K, Thompson PM, Valcour VG. Mapping white matter integrity in elderly people with HIV. Human brain mapping. 2014;35(3):97592.

18. Filippi CG, Ulu $\square$ AM, Ryan E, Ferrando SJ, van Gorp W. Diffusion tensor imaging of patients with HIV and normal-appearing white matter on MR images of the brain. American Journal of Neuroradiology. 2001;22(2):277-83. 
19. Chang L, Wong V, Nakama H, Watters M, Ramones D, Miller EN, Cloak C, Ernst T. Greater than age-related changes in brain diffusion of HIV patients after 1 year. Journal of Neuroimmune Pharmacology. 2008;3(4):265-74.

20. Gongvatana A, Schweinsburg BC, Taylor MJ, Theilmann RJ, Letendre SL, Alhassoon OM, Jacobus J, Woods SP, Jernigan TL, Ellis RJ. White matter tract injury and cognitive impairment in human immunodeficiency virusinfected individuals. Journal of neurovirology. 2009;15(2):187-95.

21. Kamat R, Brown GG, Bolden K, Fennema-Notestein C, Archibald S, Marcotte TD, Letendre SL, Ellis RJ, Woods SP, Grant I. Apathy is associated with white matter abnormalities in anterior, medial brain regions in persons with HIV infection. Journal of clinical and experimental neuropsychology. 2014;36(8):854-66.

22. Hoare J, Fouche J-P, Phillips N, Joska JA, Donald KA, Thomas K, Stein DJ. Clinical associations of white matter damage in cART-treated HIV-positive children in South Africa. Journal of neurovirology. 2015;21(2):120-8.

23. Oh SW, Shin NY, Choi JY, Lee SK, Bang MR. Altered White Matter Integrity in Human Immunodeficiency Virus-Associated Neurocognitive Disorder: A Tract-Based Spatial Statistics Study. Korean J Radiol. 2018;19(3):431-42. Epub 2018/05/02. doi: 10.3348/kjr.2018.19.3.431. PubMed PMID: 29713221; PMCID: PMC5904470.

24. Smith SM, Jenkinson M, Johansen-Berg H, Rueckert D, Nichols TE, Mackay CE, Watkins KE, Ciccarelli O, Cader MZ, Matthews PM, Behrens TE. Tract-based spatial statistics: voxelwise analysis of multi-subject diffusion data. Neuroimage. 2006;31(4):1487-505. Epub 2006/04/21. doi: 10.1016/j.neuroimage.2006.02.024. PubMed PMID: 16624579. 
25. Zhu T, Zhong J, Hu R, Tivarus M, Ekholm S, Harezlak J, Ombao H, Navia B, Cohen R, Schifitto G. Patterns of white matter injury in HIV infection after partial immune reconstitution: a DTI tract-based spatial statistics study. Journal of neurovirology. 2013;19(1):10-23.

26. Archer DB, Patten C, Coombes SA. Free-water and free-water corrected fractional anisotropy in primary and premotor corticospinal tracts in chronic stroke. Hum Brain Mapp. 2017;38(9):454662. Epub 2017/06/08. doi: 10.1002/hbm.23681. PubMed PMID: 28590584; PMCID: PMC6866851.

27. Pasternak O, Westin CF, Dahlben B, Bouix S, Kubicki M. The extent of diffusion MRI markers of neuroinflammation and white matter deterioration in chronic schizophrenia. Schizophr Res. 2015;161(1):113-8. Epub 2014/08/16. doi: 10.1016/j.schres.2014.07.031. PubMed PMID: 25126717 ; PMCID: PMC4277709.

28. Bergamino M, Kuplicki R, Victor TA, Cha YH, Paulus MP. Comparison of two different analysis approaches for DTI free $\square$ water corrected and uncorrected maps in the study of white matter microstructural integrity in individuals with depression. Human brain mapping. 2017;38(9):4690702.

29. Bergamino M, Pasternak O, Farmer M, Shenton ME, Hamilton JP. Applying a free-water correction to diffusion imaging data uncovers stress-related neural pathology in depression. NeuroImage: Clinical. 2016;10:336-42.

30. Mandl RC, Pasternak O, Cahn W, Kubicki M, Kahn RS, Shenton ME, Pol HEH. Comparing free water imaging and magnetization transfer measurements in schizophrenia. Schizophrenia research. 2015;161(1):126-32.

31. Duering M, Finsterwalder S, Baykara E, Tuladhar AM, Gesierich B, Konieczny MJ, Malik R, Franzmeier N, Ewers M, Jouvent E. Free water determines diffusion alterations and clinical status in cerebral small vessel disease. Alzheimer's \& Dementia. 2018;14(6):764-74. 
32. Albi A, Pasternak O, Minati L, Marizzoni M, Bartrés $\square$ Faz D, Bargalló N, Bosch B, Rossini PM, Marra C, Müller B. Free water elimination improves test-retest reproducibility of diffusion tensor imaging indices in the brain: a longitudinal multisite study of healthy elderly subjects. Human brain mapping. 2017;38(1):12-26.

33. Jeurissen B, Leemans A, Tournier JD, Jones DK, Sijbers J. Investigating the prevalence of complex fiber configurations in white matter tissue with diffusion magnetic resonance imaging. Hum Brain Mapp. 2013;34(11):2747-66. Epub 2012/05/23. doi: 10.1002/hbm.22099. PubMed PMID: 22611035 ; PMCID: PMC6870534.

34. Bach M, Laun FB, Leemans A, Tax CM, Biessels GJ, Stieltjes B, Maier-Hein KH. Methodological considerations on tract-based spatial statistics (TBSS). Neuroimage. 2014;100:35869.

35. Raffelt DA, Tournier JD, Smith RE, Vaughan DN, Jackson G, Ridgway GR, Connelly A. Investigating white matter fibre density and morphology using fixel-based analysis. Neuroimage. 2017;144(Pt A):58-73. Epub 2016/09/19. doi: 10.1016/j.neuroimage.2016.09.029. PubMed PMID: 27639350 ; PMCID: PMC5182031.

36. Rau Y-A, Wang S-M, Tournier J-D, Lin S-H, Lu C-S, Weng Y-H, Chen Y-L, Ng S-H, Yu SW, Wu Y-M. A longitudinal fixel-based analysis of white matter alterations in patients with Parkinson's disease. NeuroImage: Clinical. 2019;24:102098.

37. Li Y, Guo T, Guan X, Gao T, Sheng W, Zhou C, Wu J, Xuan M, Gu Q, Zhang M. Fixelbased analysis reveals fiber-specific alterations during the progression of Parkinson's disease. NeuroImage: Clinical. 2020;27:102355. 
38. Storelli L, Pagani E, Preziosa P, Filippi M, Rocca MA. Measurement of white matter fiberbundle cross-section in multiple sclerosis using diffusion-weighted imaging. Multiple Sclerosis Journal. 2020:1352458520938999.

39. Carandini T, Mancini M, Bogdan I, Rae CL, Barritt AW, Sethi A, Harrison N, Rashid W, Scarpini E, Galimberti D. Disruption of brainstem monoaminergic fibre tracts in multiple sclerosis as a putative mechanism for cognitive fatigue: a fixel-based analysis. NeuroImage: Clinical. 2021:102587.

40. Wallace EJ, Mathias JL, Ward L, Fripp J, Rose S, Pannek K. A fixel $\square$ based analysis of micro $\square$ and macro $\square$ structural changes to white matter following adult traumatic brain injury. Human brain mapping. 2020;41(8):2187-97.

41. Verhelst H, Giraldo D, Vander Linden C, Vingerhoets G, Jeurissen B, Caeyenberghs K. Cognitive training in young patients with traumatic brain injury: a fixel-based analysis.

Neurorehabilitation and neural repair. 2019;33(10):813-24.

42. Choy SW, Bagarinao E, Watanabe H, Ho ETW, Maesawa S, Mori D, Hara K, Kawabata K, Yoneyama N, Ohdake R. Changes in white matter fiber density and morphology across the adult lifespan: A cross $\square$ sectional fixel $\square$ based analysis. Human brain mapping. 2020;41(12):3198-211.

43. Jeurissen B, Tournier JD, Dhollander T, Connelly A, Sijbers J. Multi-tissue constrained spherical deconvolution for improved analysis of multi-shell diffusion MRI data. Neuroimage. 2014;103:411-26. Epub 2014/08/12. doi: 10.1016/j.neuroimage.2014.07.061. PubMed PMID: 25109526.

44. Newman BT, Dhollander T, Reynier KA, Panzer MB, Druzgal TJ. Test-retest reliability and long-term stability of three-tissue constrained spherical deconvolution methods for analyzing 
diffusion MRI data. Magn Reson Med. 2020;84(4):2161-73. Epub 2020/03/01. doi: 10.1002/mrm.28242. PubMed PMID: 32112479; PMCID: PMC7329572.

45. Gandhi NS, Moxley RT, Creighton J, Roosa HV, Skolasky RL, Selnes OA, McArthur J, Sacktor N. Comparison of scales to evaluate the progression of HIV-associated neurocognitive disorder. HIV Ther. 2010;4(3):371-9. Epub 2010/09/09. doi: 10.2217/hiv.10.23. PubMed PMID: 20824119; PMCID: PMC2933171.

46. Andersson JL, Skare S, Ashburner J. How to correct susceptibility distortions in spin-echo echo-planar images: application to diffusion tensor imaging. Neuroimage. 2003;20(2):870-88. Epub 2003/10/22. doi: 10.1016/S1053-8119(03)00336-7. PubMed PMID: 14568458.

47. Andersson JLR, Sotiropoulos SN. An integrated approach to correction for off-resonance effects and subject movement in diffusion MR imaging. Neuroimage. 2016;125:1063-78. Epub 2015/10/21. doi: 10.1016/j.neuroimage.2015.10.019. PubMed PMID: 26481672; PMCID: PMC4692656.

48. Raffelt D, Tournier JD, Fripp J, Crozier S, Connelly A, Salvado O. Symmetric diffeomorphic registration of fibre orientation distributions. Neuroimage. 2011;56(3):1171-80. Epub 2011/02/15. doi: 10.1016/j.neuroimage.2011.02.014. PubMed PMID: 21316463.

49. Tournier JD, Smith R, Raffelt D, Tabbara R, Dhollander T, Pietsch M, Christiaens D, Jeurissen B, Yeh CH, Connelly A. MRtrix3: A fast, flexible and open software framework for medical image processing and visualisation. Neuroimage. 2019;202:116137. Epub 2019/09/02. doi: 10.1016/j.neuroimage.2019.116137. PubMed PMID: 31473352.

50. Smith RE, Tournier JD, Calamante F, Connelly A. SIFT: Spherical-deconvolution informed filtering of tractograms. Neuroimage. 2013;67:298-312. Epub 2012/12/15. doi:

10.1016/j.neuroimage.2012.11.049. PubMed PMID: 23238430. 
51. Behrens TE, Woolrich MW, Jenkinson M, Johansen $\square$ Berg H, Nunes RG, Clare S, Matthews PM, Brady JM, Smith SM. Characterization and propagation of uncertainty in diffusion $\square$ weighted MR imaging. Magnetic Resonance in Medicine: An Official Journal of the International Society for Magnetic Resonance in Medicine. 2003;50(5):1077-88.

52. Dumont M, Roy M, Jodoin P-M, Morency FC, Houde J-C, Xie Z, Bauer C, Samad TA, Van Dijk KR, Goodman J. Free water in white matter differentiates MCI and AD from control subjects. Frontiers in aging neuroscience. 2019;11:270.

53. Di Tommaso P, Chatzou M, Floden EW, Barja PP, Palumbo E, Notredame C. Nextflow enables reproducible computational workflows. Nature biotechnology. 2017;35(4):316.

54. Kurtzer GM, Sochat V, Bauer MW. Singularity: Scientific containers for mobility of compute. PloS one. 2017;12(5):e0177459.

55. Benjamini Y, Hochberg Y. Controlling the false discovery rate: a practical and powerful approach to multiple testing. Journal of the Royal statistical society: series B (Methodological). 1995;57(1):289-300.

56. Raffelt DA, Smith RE, Ridgway GR, Tournier JD, Vaughan DN, Rose S, Henderson R, Connelly A. Connectivity-based fixel enhancement: Whole-brain statistical analysis of diffusion MRI measures in the presence of crossing fibres. Neuroimage. 2015;117:40-55. Epub 2015/05/26. doi: 10.1016/j.neuroimage.2015.05.039. PubMed PMID: 26004503; PMCID: PMC4528070.

57. Smith SM, Nichols TE. Threshold-free cluster enhancement: addressing problems of smoothing, threshold dependence and localisation in cluster inference. Neuroimage. 2009;44(1):8398. Epub 2008/05/27. doi: 10.1016/j.neuroimage.2008.03.061. PubMed PMID: 18501637.

58. Abraham A, Pedregosa F, Eickenberg M, Gervais P, Mueller A, Kossaifi J, Gramfort A, Thirion B, Varoquaux G. Machine learning for neuroimaging with scikit-learn. Front Neuroinform. 
2014;8:14. Epub 2014/03/07. doi: 10.3389/fninf.2014.00014. PubMed PMID: 24600388; PMCID: PMC3930868.

59. Hong S, Banks WA. Role of the immune system in HIV-associated neuroinflammation and neurocognitive implications. Brain Behav Immun. 2015;45:1-12. Epub 2014/12/03. doi:

10.1016/j.bbi.2014.10.008. PubMed PMID: 25449672; PMCID: PMC4342286.

60. Stave EA, De Bellis MD, Hooper SR, Woolley DP, Chang SK, Chen SD. Dimensions of Attention Associated With the Microstructure of Corona Radiata White Matter. J Child Neurol. 2017;32(5):458-66. Epub 2017/01/17. doi: 10.1177/0883073816685652. PubMed PMID: 28090797; PMCID: PMC5821265.

61. Luo L, Xu L, Jung R, Pearlson G, Adali T, Calhoun VD. Constrained source-based morphometry identifies structural networks associated with default mode network. Brain Connect. 2012;2(1):33-43. Epub 2012/04/04. doi: 10.1089/brain.2011.0026. PubMed PMID: 22468608; PMCID: PMC3621809.

62. Mandelkow EM, Mandelkow E. Biochemistry and cell biology of tau protein in neurofibrillary degeneration. Cold Spring Harb Perspect Med. 2012;2(7):a006247. Epub 2012/07/05. doi: 10.1101/cshperspect.a006247. PubMed PMID: 22762014; PMCID: PMC3385935.

63. Brown LA, Scarola J, Smith AJ, Sanberg PR, Tan J, Giunta B. The role of tau protein in HIVassociated neurocognitive disorders. Mol Neurodegener. 2014;9:40. Epub 2014/10/12. doi: 10.1186/1750-1326-9-40. PubMed PMID: 25304757; PMCID: PMC4210623. 


\section{Figure Legends}

Figure 1. Fiber tract-specific reductions in HIV+ compared to HIV- using whole-brain FBA. Significant fixels (FWE-corrected p-value) between HIV+ and HIV-groups displayed as the percentage decrease in the HIV+ group compared to healthy controls, displayed in coronal slices. FD: fiber density; $\log \mathrm{FC}$ : fiber bundle cross section; FDC: fiber density and cross-section.

Figure 2. Fiber tract specific $\operatorname{logFC}$ decreases in HIV infection, colored by direction. Streamlines were cropped from the template tractogram to include only significant fixels (FWE-corrected p-value $<0.05)$ for which the $\operatorname{logFC}$ metric is decreased in the HIV+ cohort compared to HIV- individuals. Significant streamlines are shown across coronal slices and colored by direction (anterior-posterior: green; superior-inferior: blue; left-right: red).

Figure 3. Fiber tract-specific significant fixels. Coronal slice showing fixels that were significantly decreased (FWE-corrected p-values) in HIV+ individuals compared to HIV-. The zoomed in area illustrates differences and p-values assigned to individual fixels in regions with crossing-fibers, around the cerebral peduncles $(\mathrm{CP})$ and middle cerebellar peduncles $(\mathrm{MCP})$. Fixels are colored by FWE corrected p-value.

Figure 4. Scatterplots show attention z-score as a function of FBA metrics: A) Fiber Density (FD), B) Fiber Cross-section $(\log \mathrm{FC})$, C) Fiber density and cross section (FDC), D) JHU white matter atlas and corresponding regions of interest. Only significant regions shown. Solid lines represent linear fit, 
and shaded areas represent the $95 \%$ confidence interval. IC: internal capsule (yellow); CR: corona radiata (teal); CR: corona radiata.

Figure 5. Group comparisons of A) FA, B) $\mathrm{FA}_{\mathrm{T}}$ C) $\mathrm{MD}_{\mathrm{T}}$ using TBSS. Statistical maps thresholded at 0.5 (not significant) show regions where $\mathrm{FA}$ and $\mathrm{FA}_{\mathrm{T}}$ are reduced in $\mathrm{HIV}+$ individuals compared to HIV-individuals. FA: Fractional Anisotropy; FA represents reduced FA or $\mathrm{FA}_{\mathrm{T}}$ in the HIV+ compared to HIV-individuals.

Figure 6. Evaluation of classification results using AdaBoost classifier. (A) Receiver operating characteristic (ROC) Curve for cognitively normal compared to cognitively impaired (CI). (B) Precision vs recall curve (PRC) for cognitively normal compared to CI. Solid line represents the mean curve using five-fold cross validation. Shaded areas represent $+/-1$ standard deviation. AUC reported as mean +/- standard deviation across five-folds. 


\section{Tables}

Table 1. Participant demographics, clinical and cognitive information

\begin{tabular}{lcccc}
\hline & & HIV+ (N=42) & HIV-(N=52) & P-value \\
\hline Age, mean(SE) & & $34.05(2.01)$ & $37.13(1.65)$ & 0.24 \\
\hline Gender, $\mathbf{n}(\boldsymbol{\%})$ & & & & \\
\hline & Female & $2(0.048)$ & $26(0.5)$ & $<\mathbf{0 . 0 0 1}$ \\
& Male & $40(0.952)$ & $26(0.5)$ &
\end{tabular}

Ethnicity, n(\%)

Hispanic or Latino

Not Hispanic or Latino
2(0.048)

40(0.952)
3(0.058)

49(0.942)

Race, n(\%)

$\begin{array}{lccl}\text { Caucasian } & 20(0.476) & 43(0.827) & \\ \text { Black.A } & 21(0.5) & 5(0.096) & <\mathbf{0 . 0 0 1} \\ \text { Other } & 1(0.024) & 2(0.038) & \\ \text { Missing } & 0(0) & 1(0.019)\end{array}$

Education, n(\%)

$\begin{array}{cccc}<12 \text { years } & 2(0.048) & 2(0.038) & \\ 12 \text { Years (HS Grad) } & 11(0.26) & 4(0.077) & \\ 12-15 \text { Years (Some College) } & 16(0.38) & 10(0.19) & \mathbf{0 . 0 0 2} \\ 16 \text { Years (Bachelor's) } & 7(0.17) & 21(0.40) & \\ >16 \text { Years (Post-Graduate) } & 6(0.14) & 15(15.52)\end{array}$

Blood Markers, mean(SE) 


\begin{tabular}{|c|c|c|c|}
\hline $\mathrm{CD} 4$ & 503.64(41.99) & - & - \\
\hline Viral Load $\left(\times 10^{3}\right)$ & $771.22(205.58)$ & - & - \\
\hline Neurofilament (NfL) & $11.39(1.91)$ & - & - \\
\hline Tau & $2.47(1.07)$ & - & - \\
\hline
\end{tabular}

\section{Cognitive Score}

\begin{tabular}{cccc}
\hline Total Summary Score & $-1.45(0.386)$ & $0.325(0.444)$ & $\mathbf{0 . 0 3 5}$ \\
Executive Z-Score & $-0.122(0.083)$ & $0.227(0.108)$ & $>0.05$ \\
Attention Z-Score & $-0.294(0.101)$ & $0.130(0.092)$ & $\mathbf{0 . 0 3 3}$ \\
Motor Z-Score & $-0.208(0.097)$ & $0.246(0.086)$ & $\mathbf{0 . 0 1 6}$ \\
Learning Z-Score & $-0.309(0.082)$ & $-0.153(0.100)$ & $>0.05$ \\
Speed of Learning Z-Score & $-0.188(0.091)$ & $0.042(0.096)$ & $>0.05$ \\
Memory Z-Score & $-0.261(0.096)$ & $-0.223(0.102)$ & $>0.05$ \\
Verbal Z-Score & $-0.073(0.086)$ & $0.057(0.106)$ & $>0.05$ \\
\hline
\end{tabular}

Note: Continuous variables summarized as Mean(SE), categorical variables are summarized as frequency(percentages). CD4 in cells $/ \mathrm{mm}^{3}$, NfL in $\mathrm{pg} / \mathrm{ml}$. Significant results are shown as bold. 
Table 2. Linear regression model comparing the FBA metrics in HIV+ and HIV- individuals, with age and sex included as covariates.

ROI HIV + HIV - $\quad$ Estimate $\underset{\begin{array}{c}\text { Std } \\ \text { Error }\end{array} p \text {-value }}{\begin{array}{c}\text { Effect } \\ \text { Size }\end{array}}$

\section{FD}

Right

PLIC

0.601

$0.614-0.020$

0.006

0.03

0.46

Left PLIC $\quad 0.610$

$0.619-0.013$

0.007

0.28

0.29

Left SCR

0.352

$0.356-0.004$

0.003

0.70

0.27

Right

SCR

0.352

$0.355 \quad-0.001$

0.004

0.81

0.17

Right

ICP

0.402

$0.406 \quad 0.007$

0.012

0.81

0.08

Left ICP

0.427

0.427

0.012

0.011

0.72

0.01

MCP

0.448

0.443

0.004

0.009

0.81

0.14

\section{$\log F C$}

Right

PLIC

$-0.032$

$\begin{array}{ll}-0.007 & -0.069\end{array}$

0.019

$<0.001$

0.30

Left PLIC $\quad-0.028$

$-0.011 \quad-0.067$

0.019

$<0.001$

0.19

Left SCR

0.025

0.023

$-0.043$

0.019

$\mathbf{0 . 0 3}$

0.03

Right

SCR

0.026

$0.029-0.046$

0.019

0.03

0.04

Right

ICP

$-0.017$

$0.017 \quad-0.077$

0.017

$<0.001$

0.43

Left ICP

$-0.013$

$0.024-0.075$

0.016

$<0.001$

0.46

MCP

0.068

$0.110 \quad-0.094$

0.021

$<0.001$

0.40 


\section{FDC}

\begin{tabular}{lcccccc}
$\begin{array}{l}\text { Right } \\
\text { PLIC }\end{array}$ & 0.585 & 0.617 & -0.065 & 0.015 & $<\mathbf{0 . 0 0 1}$ & 0.45 \\
Left PLIC & 0.599 & 0.621 & -0.059 & 0.015 & $<\mathbf{0 . 0 0 1}$ & 0.32 \\
Left SCR & 0.361 & 0.367 & -0.023 & 0.009 & $\mathbf{0 . 0 2}$ & 0.15 \\
Right & 0.362 & 0.368 & -0.020 & 0.010 & 0.06 & 0.16 \\
SCR & 0.396 & 0.415 & -0.022 & 0.129 & 0.11 & 0.36 \\
$\begin{array}{l}\text { Right } \\
\text { ICP }\end{array}$ & 0.420 & 0.434 & -0.019 & 0.012 & 0.12 & 0.27 \\
Left ICP & 0.485 & 0.502 & -0.043 & 0.016 & $\mathbf{0 . 0 2}$ & 0.24 \\
MCP & & & & & & \\
\hline
\end{tabular}

Note: Estimate is average difference between HIV+ and HIV-for which HIV-is taken as the reference group (two-tailed t-test, FDR corrected at the $\alpha=0.05$ significance level). PLIC: posterior limb of Internal Capsule, SCR: Superior Corona Radiata, ICP: inferior cerebellar peduncle MCP: Middle Cerebellar Peduncle. Significant results are shown as bold. 
Table 3. Precision, recall, and F1-scores for 5 different classifiers for FBA and fwcDTI metrics reported using five-fold cross validation in HIV+ subjects only.

\begin{tabular}{lcccc}
\hline Classifiers & Metrics & Precision & Recall & F-Score \\
\hline LDA & FBA & 0.62 & 0.60 & 0.58 \\
& fwcDTI & 0.50 & 0.43 & 0.46 \\
Random & FBA & 0.62 & 0.77 & 0.76 \\
$\begin{array}{l}\text { Forest } \\
\text { fwcDTI }\end{array}$ & 0.53 & 0.53 & 0.53 \\
\hline NaiveBayes & FBA & 0.62 & 0.58 & 0.55 \\
& fwcDTI & 0.38 & 0.38 & 0.38 \\
\hline & FBA & $\mathbf{0 . 8 0}$ & $\mathbf{0 . 7 7}$ & $\mathbf{0 . 7 6}$ \\
\hline AdaBoost & fwcDTI & 0.48 & 0.46 & 0.47 \\
\hline
\end{tabular}

Note: Scores reported as weighted average across 3 classes. FBA: Fixel-based analysis; fwcDTI: free water corrected diffusion tensor imaging; LDA: Linear Discriminant Analysis. 


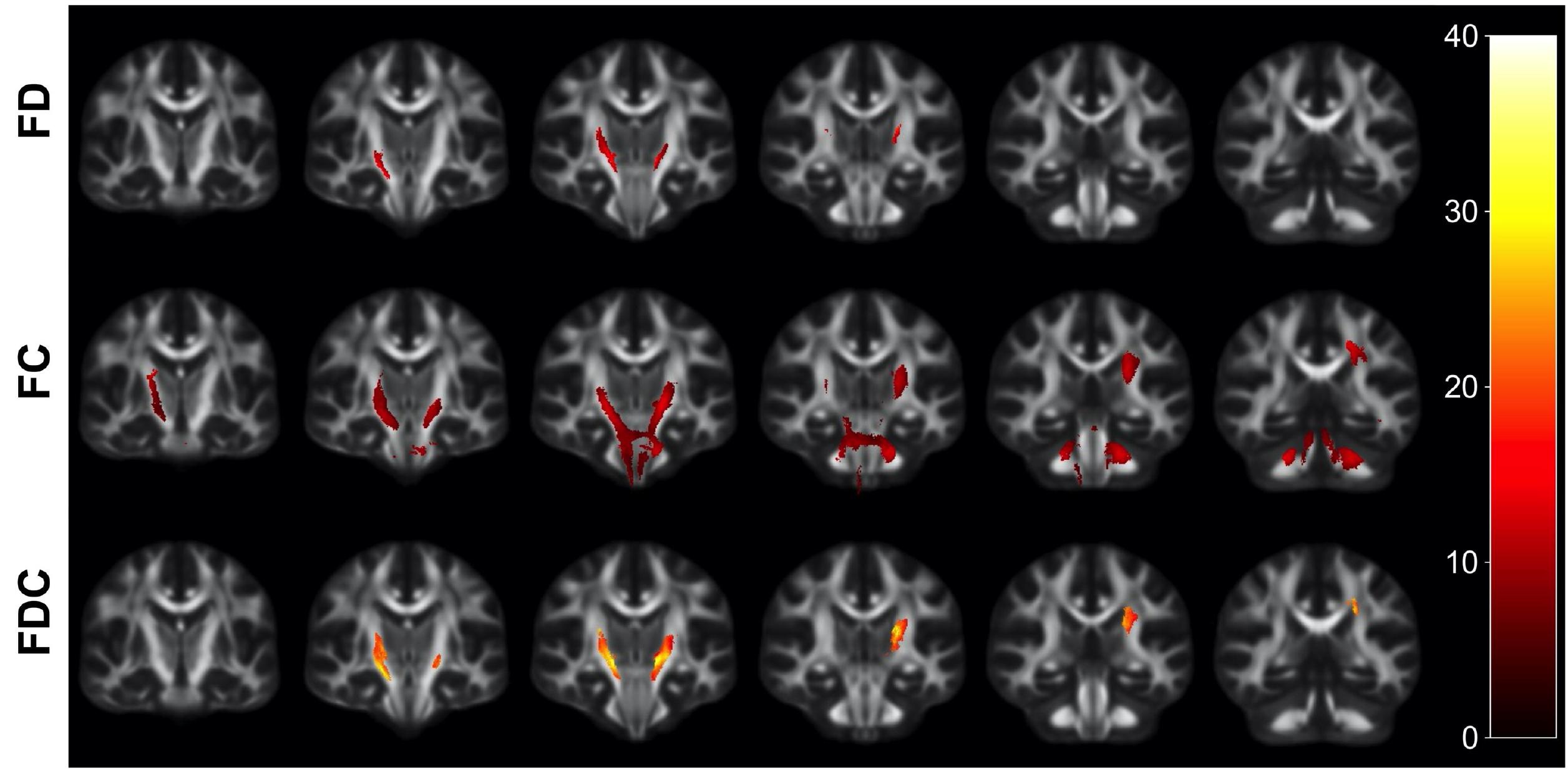




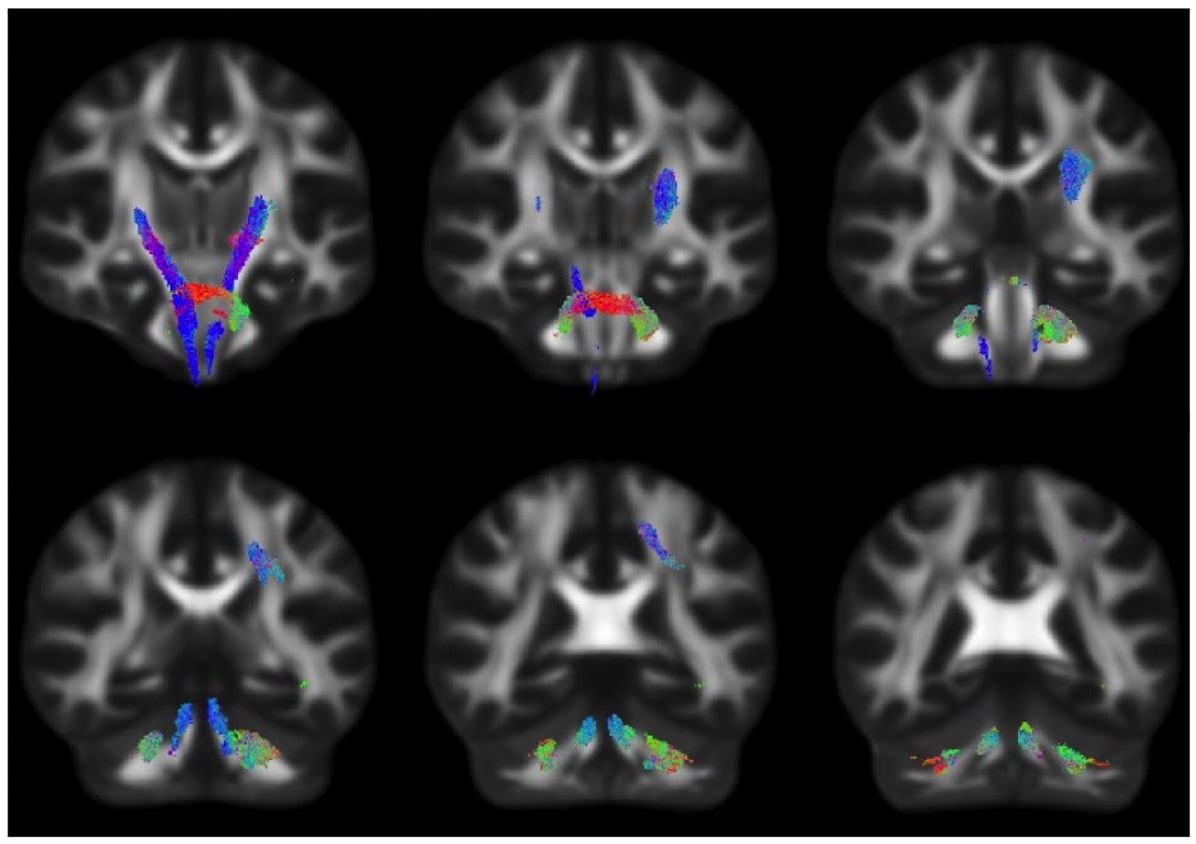




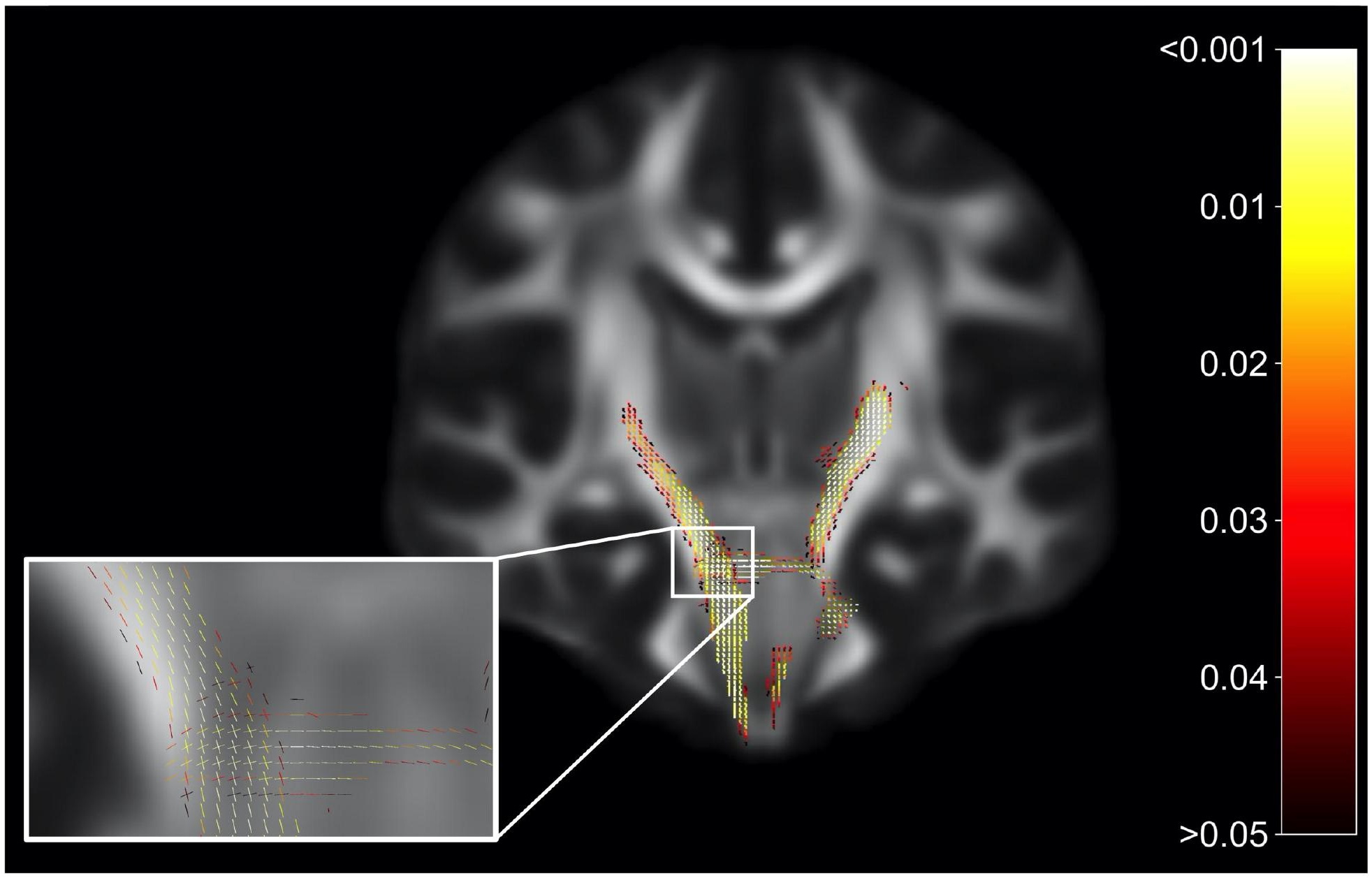


